\title{
Development of the Measurement System for the Search of an Electric Dipole Moment of the Electron with Laser-Cooled Fran- cium Atoms
}

T. Inoue ${ }^{1, a}$, S. Ando ${ }^{1}$, T. Aoki ${ }^{1}$, H. Arikawa ${ }^{1}$, S. Ezure ${ }^{1}$, K. Harada ${ }^{1}$, T. Hayamizu ${ }^{1}$, T. Ishikawa ${ }^{1}$, M. Itoh ${ }^{1}$, K. Kato ${ }^{1}$, T. Kato ${ }^{1, b}$, H. Kawamura ${ }^{1}$, H.S. Nataraj ${ }^{1, c}$, T. Sato ${ }^{1, d}$, A. Uchiyama ${ }^{1}$, T. Aoki ${ }^{2}$, T. Furukawa ${ }^{3}$, A. Hatakeyama ${ }^{4}$, K. Hatanaka ${ }^{5}$, K. Imai ${ }^{6}$, T. Murakami ${ }^{7}$, Y. Shimizu ${ }^{8}$, T. Wakasa ${ }^{9}$, H.P. Yoshida ${ }^{5}$, and Y. Sakemi ${ }^{1}$

${ }^{1}$ Cyclotron and Radioisotope Center, Tohoku University, Miyagi, Japan

${ }^{2}$ Graduate School of Arts and Sciences, University of Tokyo, Tokyo, Japan

${ }^{3}$ Department of Physics, Tokyo Metropolitan University, Tokyo, Japan

${ }^{4}$ Department of Applied Physics, Tokyo University of Agriculture and Technology, Tokyo, Japan

${ }^{5}$ Research Center for Nuclear Physics, Osaka University, Osaka, Japan

${ }^{6}$ Advanced Science Research Center, Japan Atomic Energy Agency, Ibaraki, Japan

${ }^{7}$ Department of Physics, Kyoto University, Kyoto, Japan

${ }^{8}$ Department of Physics, Tohoku University, Miyagi, Japan

${ }^{9}$ Department of Physics, Kyushu University, Fukuoka, Japan

\begin{abstract}
We plan to measure the permanent electric dipole moment (EDM) of the electron, which has the sensitivity to the $\mathrm{CP}$ violation in theories beyond the standard model by using the laser-cooled francium (Fr) atom. This paper reports the present status of the EDM measurement system. A high voltage application system was constructed in order to produce the strong electric field $(100 \mathrm{kV} / \mathrm{cm})$ needed for the experiment. After conditioning, the leakage current was $10 \mathrm{pA}$ when a high voltage of $43 \mathrm{kV}$ was applied. Also, a drift of an environmental field was measured at the planned location of the Fr-EDM experiment. The drift is suppressed at present down to the level of $10 \mathrm{pT}$ by installing a 4-layer magnetic shield. Improvements are still needed to reach the required field stability of $1 \mathrm{fT}$.
\end{abstract}

\section{Introduction}

Permanent electric dipole moment (EDM) of a particle is an important observable in searching for physics beyond the Standard Model (SM) of elementary particle physics [1]. A finite value of the EDM associated with spin constitutes clear evidence of the violation of the time-reversal symmetry, which is equivalent to the $\mathrm{CP}$ violation through the $\mathrm{CPT}$ theorem. The EDM due to the $\mathrm{CP}$-violating

\footnotetext{
ae-mail: inoue_t@cyric.tohoku.ac.jp

${ }^{\mathrm{b}}$ Present address: Progress Technologies Inc.

${ }^{\mathrm{c}}$ Present address: Department of Physics, Indian Institute of Technology Roorkee, Uttarakhand, India

${ }^{\mathrm{d}}$ Present address: Department of Physics, Tokyo Institute of Technology, Tokyo, Japan
} 
phase in the SM, as observed in the $\mathrm{CP}$-nonconserving decays of $\mathrm{K}$ and $\mathrm{B}$ mesons, is too small to be detectable. On the other hand, most theories beyond the SM predict a finite value of the EDM, which can be detected with modern experimental techniques. Thus, the search for EDM constitutes a stringent test that discriminates between the SM and theories beyond it. Actually, for example, the latest experimental upper limits for the neutron EDM [2] and the electron EDM [3] already enter the region predicted by Supersymmetry, which is one of the candidate theoretical models beyond the SM. In this respect, EDM searches have already stepped into the phase in which the experimental upper limits would urge the theories to modify their parameters.

A paramagnetic atom and a polar molecule have been subjects of the electron EDM search, since they have certain mechanisms to enhance the electron EDM. The francium (Fr) atom, which is one of the paramagnetic atoms, has the largest enhancement factor in the alkali atoms [4]. The search for the electron EDM has been done using atomic or molecular beams. However, in these experiments, the statistical and systematic errors are limited due to the short coherence time and the effect of the motional magnetic field. In order to overcome these limitations, the laser-cooling technique is utilized [5]. Advantages of the laser-cooled atom for the EDM search are as follows. Since the lasercooled atoms can be trapped under a high vacuum environment, a long coherence time is realized. The velocity of the trapped atom is small, and consequently the motional magnetic field is suppressed. Since the Fr atom is an alkali atom, it is a suitable system in which to apply the laser-cooling technique. In view of these advantages, we plan to perform the EDM search experiment using the laser-cooled $\mathrm{Fr}$ atom. Internationally, there are several experiments in progress using the Fr atoms at various locations including ISOLDE in Geneva, TRIUMF in Vancouver and LNL in Padova. ISOLDE can supply the high-intensity Fr ion beam more than $10^{9}$ ions/sec through a proton-induced spallation reaction [6]. The experiments searching for the atomic parity non-conservation (PNC) by using Fr are individually being planned at TRIUMF and LNL. They have already trapped the Fr atoms by using the Magneto-Optical Trap (MOT). The numbers of trapped Fr atoms are $10^{5}$ at TRIUMF [7] and $10^{4}$ at LNL [8], respectively. The experiments searching for the violation of the fundamental symmetries such as the measurements of EDM and PNC require several appropriate experimental conditions and sufficient beam times. It is the Cyclotron and Radioisotope Center (CYRIC) at Tohoku University, a restively small facility, which comes in as the facility satisfying these requirements. Our laser-cooled Fr factory, which is being constructed at CYRIC, consists of the beam swinger magnet, the Fr ion source, the beam transport system, the ion-to-neutral atom converter, the MOT system, and the EDM measurement system. H. Kawamura et al. report the detail of our factory in these proceedings. In this paper, we report the present status of the high voltage application system and the result of an environmental magnetic field measurement around the future location of the EDM experiment, both important items for the EDM measurement system.

\section{High Voltage Application}

The application of a high electric field $(100 \mathrm{kV} / \mathrm{cm})$ is one of the key factors in the EDM search experiment, since the EDM is experimentally deduced from a tiny change of the spin precession frequency upon the reversal of an applied electric field along a magnetic field. A high voltage system is under development. A schematic view of the developed high voltage system is presented in Figure 1 (a). We employ the oxygen free copper as a material for the electrodes. The size of each cylindrical electrode is $2 \mathrm{~cm}$ in diameter and $2 \mathrm{~cm}$ long with a machining accuracy of $0.2 \mathrm{~mm}$. The gap between the electrodes is fixed to be $1 \mathrm{~cm}$ by an insulator made of ceramic material. The electrodes, covered by the insulator, were placed inside a chamber with a vacuum pressure of $7 \times 10^{-6} \mathrm{~Pa}$. A leakage current across the electrodes was measured with a picoammeter. The picoammeter was protected against sudden discharge by inserting a low-pass filter. 
(a)

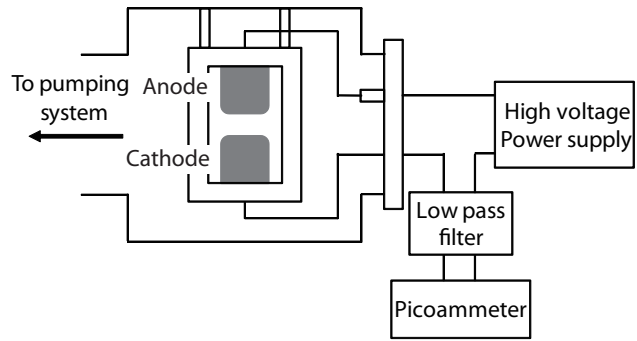

(b)

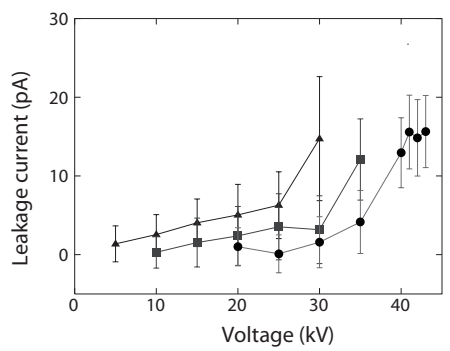

Figure 1. (a) Schematic view of the high-voltage system. (b) Applied voltage dependence of the leakage current. The triangles show the leakage current measured with no conditioning, while the squares and circles show it after the $10 \mathrm{kV}$ conditioning for 3 days and $20 \mathrm{kV}$ for 1.5 days, respectively. Error bars show the standard error.

In Figure 1 (b), the applied voltage dependences of the leakage current are plotted. Without treatment of the electrode surface, such as polishing, the discharge occurred at a voltage of $30 \mathrm{kV}$. In order to extend the discharge voltage, conditioning was applied. Conditioning is a method based on glow discharge that destroys sharp points and dust on the surface of the electrode. First, the voltage of $10 \mathrm{kV}$ was applied for 3 days, and then the discharge voltage was extended to $35 \mathrm{kV}$. Next, the application of $20 \mathrm{kV}$ was performed for 1.5 days. The conditioning resulted in the extension of the discharge voltage up to $43 \mathrm{kV}$ and the leakage current of $15 \mathrm{pA}$. Until now, we have applied the voltage of $50 \mathrm{kV}$ without the discharge for 3 minutes without the leakage current measurement.

\section{Environmental field measurement}

The stability of the magnetic field around the apparatus is the most important factor for any EDM search experiments. Generally, the change of $1 \mathrm{nT}$ in the magnetic field induces a shift of about $10 \mathrm{~Hz}$ in the spin precession frequency of a paramagnetic atom. On the other hand, for the applied electric field of $100 \mathrm{kV} / \mathrm{cm}$ and the enhancement factor $R \sim 895$ of Fr [4], the electron EDM of magnitude $|d|=10^{-28} \mathrm{ecm}$ gives a frequency change of less than $1 \mu \mathrm{Hz}$. We measured the drift of the environmental field at the planned location of the EDM experiment to optimize the design of a suitable magnetic shield. In order to suppress the change of the magnetic field at the EDM measurement location, we tentatively introduced a four layer cylindrical magnetic shield that was constructed for the development of the highly sensitive magnetometer utilizing the nonlinear magneto-optical rotation (NMOR) effect in $\mathrm{Rb}$ atomic vapor [9].

The field measurements were performed by using fluxgate magnetometers when the AVF cyclotron at CYRIC was on or off. The direction of the measured fields was the same as that of the gravity. Figure 2 shows drifts of the environmental field (a) and the residual field inside the magnetic shield (b). It should be pointed out that the direction of the residual field is opposite to that of the environmental field. This suggests that the source of the residual field inside the shield mainly comes from a residual magnetization of the shield itself. When the cyclotron was operated, a shift of both environmental and residual fields occurred. Additionally, sudden jumps of the environmental field were observed, while they were suppressed in the residual field. The movement of the cars, which stopped outside the experimental room, might induced these sudden changes. Short term stabilities of the residual fields with and without the cyclotron operation are comparable. In this situation, the Allan deviation of the residual field with the cyclotron operation is about $20 \mathrm{pT}$ for an integration time 

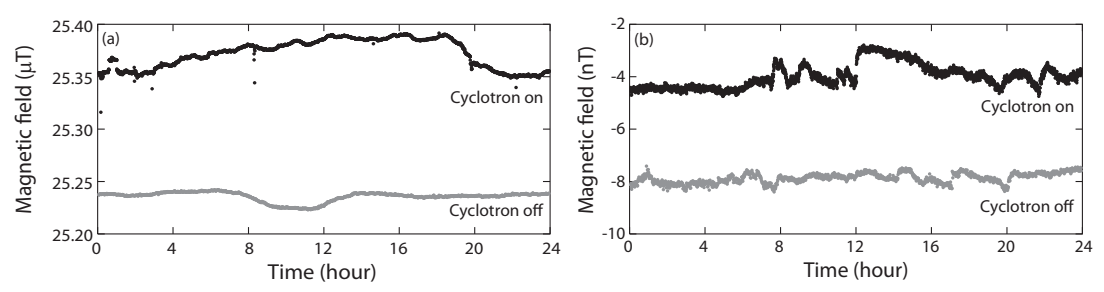

Figure 2. Drifts of the environmental fields (a) and the residual fields in the magnetic shield (b). The black dots show the fields measured during the cyclotron operation, while the gray dots show them with the cyclotron unoperated.

of $1 \mathrm{~s}$, which is much larger than the required field stability of $1 \sim 0.1 \mathrm{fT}$. In order to overcome this gap, we plan to construct a magnetic field stabilization system [10] based on NMOR effect, which can reach the shot-noise-limited sensitivity of $0.3 \mathrm{fT} / \sqrt{\mathrm{Hz}}[11]$.

\section{Summary}

The high voltage application system was constructed for the EDM search experiment with lasercooled francium atoms. The application of $43 \mathrm{kV}$ was achieved after conditioning. In order to reach $100 \mathrm{kV}$, some developments are in progress, for example, the conditioning to higher voltage for longer time. Also, the magnetic field stability was investigated when the AVF cyclotron was operated, since the change of magnetic field is the main source of a false EDM signal. By introducing a cylindrical magnetic shield, the field stability of $20 \mathrm{pT}$ for the integration time of $1 \mathrm{~s}$ was achieved. To reach the required stability of the magnetic field, a field stabilization system will be constructed and a highly sensitive magnetometer will be introduced.

\section{Acknowledgements}

The authors would like to thank A. Yoshimi for lending the magnetic shield. This research is partially supported by Grant-in-Aid for Scientific Research (No. 21104005) from the Ministry of Education, Culture, Sports, Science, and Technology, Japan.

\section{References}

[1] I.B. Kriplovich, S.K. Lamoreaux, CP Violation Without Strangeness, Springer, Heidelberg, 1997.

[2] C.A. Baker et al., Phys. Rev. Lett. 97, 131801 (2006).

[3] J.J. Hudson et al., Nature. 473, 493-496 (2011).

[4] D. Mukherjee et al., J. Phys. Chem. A 113, 12549-12557 (2009).

[5] C. Chin et al., Phys. Rev. A 63, 033401 (2001).

[6] A. Coc et al., Phys. Lett. 163B, 66-70 (1985).

[7] S. Aubin et al., AIP Conf. Proc. 1525, 530 (2013) and Vive la Francium | Joint Quantum Institute, http://jqi.umd.edu/news/vive-la-francium

[8] C. de Mauro et al., Phys. Rev. A 78, 063415 (2008).

[9] A. Yoshimi et al., Phys. Procedia. 17, 245-250 (2011).

[10] T. Bryś et al., Nucl. Instrum. Methods Phys. Research A 554, 527-539 (2005).

[11] D. Budker et al., Phys. Rev. A 62, 043403 (2000). 